\title{
An Italian Model for the Management of Cancer Patients during COVID-19 Pandemic: The Regional Center for Oncological Orientation (COrO) of Taranto (ROP)
}

\author{
Salvatore Pisconti ${ }^{a}$ Gabriella Modoni ${ }^{a}$ Concetta Cafiero ${ }^{a} \quad$ Giuseppe Simeone $^{a}$ \\ Giammarco Surico $^{\text {b }}$ Giovanni Gorgonic

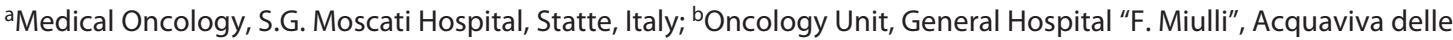 \\ Fonti, Italy; 'Department of Health, Puglia Region, Social Welfare and Sport, Bari, Italy
}

\section{Highlights of the Study}

- Novel management of cancer patients during the COVID-19 pandemic requires close collaboration between private centers and hospitals.

- To date, management guidelines for cancer patients in pandemics are not available.

- Our strategy was to minimize any interruption of cancer treatment through the extension of Taranto's Health Authority Oncology Network (COrO).

\section{Keywords}

Management · Cancer patients · COVID-19 · Regional Center for Oncological Orientation

\begin{abstract}
Objective: The recent outbreak of COVID-19 limited the resources of the National Health System necessitating the formulation of novel practice recommendations for oncological care. To date, management guidelines for cancer patients in case of pandemic are not available. Each center tried to manage its own needs and requests independently, often reducing access to treatment and diagnostic exams to patients. Here, we have described the management of cancer patients during COVID-19 infection with suggestions of some practical approaches applied by our Regional Center for Oncological Orientation (COrO) in S.G. Moscati Hospital (Taranto, Italy). Subjects and Methods: Our strategy was to
\end{abstract}

karger@karger.com www.karger.com/mpp

Karger $\stackrel{\text { ' }}{5}$
(C) 2021 The Author(s).

Published by S. Karger AG, Basel

This is an Open Access article licensed under the Creative Commons Attribution-NonCommercial-4.0 International License (CC BY-NC) (http://www.karger.com/Services/OpenAccessLicense), applicable to the online version of the article only. Usage and distribution for commercial purposes requires written permission. minimize any interruption of cancer treatment through the extension of Taranto's Health Regional (COrO). The extension of the oncological network, assisted by the General Management of Taranto ASL through agreements with private structures in Taranto's area, allowed cancer patients to receive up to 11 different types of services, according to their needs (first investigation or follow-up), and represents an exclusive organization on the entire Italian territory. Results: Thanks to the organization of the COrO in 2020, 1,406 first oncological visits and 566 preparatory treatments were carried out, 372 of exemption for oncological pathology (free health care) were activated, and 1,742 instrumental investigations and 7 cases of civil invalidity were performed (certificate of disability). Conclusions: We have overcome barriers to care of oncology patients leading to a reduction of waiting lists representing a practical application model that can be extended to other healthcare settings.

(c) 2021 The Author(s).

Published by S. Karger AG, Basel

Correspondence to:

Concetta Cafiero, concetta.cafiero@ asl.taranto.it 


\section{Introduction}

In December 2019, an outbreak of atypical pneumonia, caused by the single-stranded positive RNA virus SARS-CoV-2, known as COVID-19, and characterized by rapid human to human transmission, was identified at Wuhan (China) [1]. This viral disease was identified as a public health emergency of major international concern and was declared a pandemic by the WHO [2]. Italy was found unprepared to manage lockdown patients with chronic diseases, due to limited availability of infrastructure and resources needed to address a large-scale emergency. With the WHO declaring the novel coronavirus outbreak a pandemic, there was an urgent need to address the impact of such a pandemic on cancer patients. Many cancer patients frequently visit the hospital for treatment and disease surveillance $[3,4]$, and they may be immunocompromised due to the underlying malignancy or anticancer therapy and are at higher risk of developing infections. During the COVID-19 pandemic, many medical centers experienced cancelations and postponements of in-person outpatient medical visits [5], and out of necessity, the use of telemedicine and virtual care was suggested as a method to maintain a continuum of healthcare for patients [6]. Telemedicine has been tested in multiple clinical settings, demonstrating at least equivalency to inperson care and high levels of patient and health professional satisfaction [7]. Telemedicine, by minimizing inperson visits, played an important role in many medical centers because it reduced the transmission of the virus and protected medical professionals from infection [810]. Telemedicine, carried out in our organization by the specialist's office, is critical to cancer care [11] but unfortunately not sufficient to manage oncological patients, due to the necessity of numerous clinical instrumental examination useful for the follow-up therapy.

Here, we describe the approach to the management of cancer patients developed by our Regional Center for Oncological Orientation (COrO) in S.G. Moscati Hospital (Taranto-Italy) in the pandemic 2020 year and in particular during both the first wave (characterized by a long lockdown period in February and March 2020) and the second wave (from September to December 2020) of the epidemic.

\section{Materials and Methods}

A primary challenge of the pandemic was resource management since the priority of many hospitals was the treatment of COVID-19 patients. The pandemic emergency led to an improvised reorganization of the structures, of the diagnosis and treat-

Italian Model for the Management of

Cancer Patients during COVID-19 ment processes, and the services and healthcare personnel intended to deal exclusively with the care of patients affected by the coronavirus in order to limit the infection as much as possible. This situation led to inevitable consequences in oncology. The emergency brought out strong critical issues that also derived from the nonoptimal interaction and integration between hospital and local medicine in the process of taking charge of the cancer patient who presents the complexity of a clinical, organizational, managerial, and economic nature. In this scenario, the Oncology Network represents a possible solution because it was born and developed precisely in response to the need to overcome the problem of fragmentation in the provision of health services and to respond to the needs of citizens by guaranteeing continuity of care between hospital and catchment area. The COrO of S.G. Moscati Hospital of Statte (Taranto, Italy) is part of the Apulian Oncological Network (Rete Oncologica Pugliese ROP), created to meet the needs of cancer patients through the creation of a global care model starting from the first oncologic visit, booked or from attending physician or from oncologist or from the surgeon. The COrO after the onset of the pandemic made efforts to reorganize its services for better management of cancer patients during the state of emergency. The center is composed of coordinating, medical, nursing, and administrative staff. In particular, the COrO takes care of booking specialists' visits and tests with waiting times from $24 \mathrm{~h}$ to a week as well as for follow-ups. It prepares requests to the diagnostic-therapeutic path and compiles and updates medical records. In addition, this organization releases exemption tickets for neoplastic pathology, takes care of the management of paper and computer documentation, and reports patients to the corporate Interdisciplinary Pathology Group for collective discussion. The whole process is carried out following the Guidelines of the National System of the National Institutes of Health.

Our strategy was to minimize any interruption of cancer treatment through the extension of the hospital network of Taranto's Health Regional (COrO) aimed at reducing (1) the workload in the public hospitalization facilities and (2) the waiting lists for instrumental clinical examinations, such as bone densitometry, breast ultrasound, mammography, abdominal ultrasound, chest X-ray, CT-scan, pelvic ultrasound, magnetic resonance, scintigraphy, colonoscopy, and gastroscopy. The extension provides the subdivision of clinical instrumental examination only for oncological patients over 9 accredited private hospitals scattered throughout the Taranto's greater area (Table 1). The extension of the oncological network, through the private territorial health structures, allowed cancer patients to receive up to 11 different types of services, according to their needs, and represents a unique management model throughout the Italian national territory. This allowed us to perform not only diagnostic tests but also organize first visits, evaluate the disease, and prepare therapy in close collaboration with oncologists.

\section{Results}

Table 1 shows all the clinical instrumental examinations for oncological patients in 9 accredited private hospitals scattered over the territory of Taranto. This 
Table 1. Extension of Taranto's oncological orientation region center (COrO)

\begin{tabular}{|c|c|c|c|c|c|c|c|c|c|}
\hline & Clinic \#1 & Clinic \#2 & Clinic \#3 & Clinic \#4 & Clinic \#5 & Clinic \#6 & Clinic \#7 & Clinic \#8 & Clinic \#9 \\
\hline Bone densitometry (520) & 174 & 173 & 173 & & & & & & \\
\hline Breast ultrasound (520) & 173 & & & 173 & 174 & & & & \\
\hline Abdominal ultrasound (624) & 104 & 104 & 104 & 104 & 104 & 104 & & & \\
\hline Chest X-ray (624) & 104 & 104 & 104 & 104 & 104 & & & 104 & \\
\hline CT-scan (total body) $(1,248)$ & 250 & 250 & 250 & 249 & 249 & & & & \\
\hline Magnetic resonance (156) & 52 & 52 & & & 52 & & & & \\
\hline Scintigraphy (104) & & & & & & & & & 104 \\
\hline Colonoscopy (52) & & & & 26 & 26 & & & & \\
\hline Gastroscopy (52) & & & 17 & 18 & 17 & & & & \\
\hline
\end{tabular}

The extension provides the subdivision of clinical instrumental examination only for oncological patients over 9 accredited private hospitals scattered throughout the Taranto's area.

Table 2. Activity of COrO during 2019 and the activity of COrO during the COVID-19 pandemic in 2020

\begin{tabular}{llllll}
\hline & First visit & $\begin{array}{l}\text { Completions } \\
\text { preparatory to } \\
\text { therapy }\end{array}$ & $\begin{array}{l}\text { Activation exemption } \\
\text { for pathology (free care) }\end{array}$ & $\begin{array}{l}\text { Instrumental } \\
\text { investigations }\end{array}$ & $\begin{array}{l}\text { Civil invalidity } \\
\text { (certificate of } \\
\text { disability) }\end{array}$ \\
\hline Year: 2019 & 1,285 & 450 & 187 & 1,387 & 1 \\
Total & & & & & 176 \\
\hline Year: 2020 & 138 & 61 & 37 & 176 & 3 \\
January & 126 & 39 & 32 & 87 & 1 \\
February & 84 & 26 & 24 & 108 & 0 \\
March & 109 & 31 & 35 & 198 & 0 \\
April & 113 & 52 & 24 & 119 & 1 \\
May & 103 & 54 & 29 & 175 & 0 \\
June & 118 & 48 & 30 & 115 & 0 \\
July & 99 & 53 & 22 & 126 & 0 \\
August & 121 & 55 & 37 & 187 & 0 \\
September & 158 & 45 & 47 & 142 & 0 \\
October & 115 & 57 & 27 & 193 & 0 \\
November & 122 & 45 & 28 & 1,742 & 7 \\
December & 1,406 & 566 & 372 & & \\
\hline Total & & & & & \\
\hline
\end{tabular}

organization ensured that the diagnosis, evaluation of the disease, and timely treatment of cancer patients were not compromised during the SARS-CoV-2 pandemic. The management of these patients was adapted to the best resources available in the area of Taranto. A network of hospitals (public and private) was created in order to eliminate the waiting lists for cancer diseases; this organization allowed examinations indispensable for the diagnosis to be performed rapidly. The evalua- tion of the disease and treatment preparation in cancer patients even exceeded the number of examinations and services carried out through the COrO in 2019, a year in which there was no pandemic and in which only public resources were used (Table 2). In the first year of the pandemic 2020, 1,406 first oncological visits and 566 completions preparatory to therapy were carried out, 372 of exemption for oncological pathology (free health care for 372 patients) were activated, 1,742 clin- 
ical exams were booked, and 7 cases of civil invalidity (certificate of disability for 7 patients) were performed. This organization of the public network with the private sector led to a reduction of waiting lists for cancer patients, so much so that it could be used as a model even in periods of nonpandemic to reduce waiting periods.

\section{Discussion}

The recent outbreak of COVID-19 has enabled cancer patients to choose whether to continue with their treatment or to interrupt it for fear of infection. Moreover, the pandemic limited the resources of the public health system and necessitated the formulation of novel practice recommendations for oncological care. To date, management guidelines for hospitalized cancer patients during the pandemic are not available. Only in a few cases has the scientific literature indicated suggestions for the management and care of the cancer patients in this pandemic phase. For example, the Italian Association of Medical Oncology (Associazione Italiana Oncologia Medica AIOM) strongly suggests extending continuity of care outside hospitals, activating regional oncology networks, and using telemedicine [12]. Undoubtedly, it is premature to make judgments on the "response" to the pandemic on our part, also considering the lack of a fundamental element such as the application of telemedicine widely used in other healthcare contexts [13]. However, in this regard, it is necessary to emphasize that the "response" given to the health emergency in the various Italian regions has been conditioned, in a decisive way, by the previous organization of the National Health System. In this perspective, the management of cancer patients during COVID-19 infection by our COrO, assisted by the General Management of Taranto ASL through agreements with private institutions in the area, represents a practical application model that can be extended, with appropriate improvements and implementations to other health services.

\section{Conclusion}

The recent pandemic has negatively impacted the care of particularly vulnerable cancer patients. We have outlined our clinical experience in managing cancer patients in the COVID-19 era and also our ability to overcome the barriers to care of oncology patients. Our strategies were to minimize any interruption to cancer treatment through the extension of Taranto's Health Authority Oncology Network (COrO) aimed at reducing the workload of public hospitalization facilities and of course the waiting lists for instrumental clinical examinations representing an exclusive organization on the entire Italian territory.

\section{Statement of Ethics}

All procedures were conducted according to the principles expressed in the Declaration of Helsinki and the Guideline for Good Clinical Practice.

\section{Conflict of Interest Statement}

The authors have no conflicts of interest to declare.

\section{Funding Sources}

This research did not receive any financial support from funding agencies in the public, commercial, or not-for-profit sectors.

\section{Data Availability Statement}

The data are available.

\section{Author Contributions}

C.C., S.P., G.M., and G.S. contributed to conceptualization; S.P., G.S., and G.G. contributed to methodology and supervision; G.M. and S.P. contributed to project administration. C.C. and G.S. contributed to data collection and analysis. All authors read and approved the final manuscript.

\section{References}

Italian Model for the Management of Cancer Patients during COVID-19
1 Krishnan A, Hamilton JP, Alqahtani SA, Woreta TA. A narrative review of coronavirus disease 2019 (COVID-19): clinical, epidemiological characteristics, and systemic manifestations. Intern Emerg Med. 2021 Jan 16:1-16.

2 CiottiM, Angeletti S, Minieri M, Giovannetti M, Benvenuto D, Stefano Pascarella S, et al. COVID-19 outbreak: an overview. Chemotherapy. 2019;64:215-23.
3 Tsamakis K, Gavriatopoulou M, SchizasD, Stravodimou A, Mougkou A, Tsiptsios D, et al. Oncology during the COVID-19 pandemic: challenges, dilemmas and the psychosocial impact on cancer patients. Oncol Lett. 2020 Jul;20:441-7. 
4 Al-Shamsi HO, Alhazzani W, Alhuraiji A, Coomes EA, Chemaly RF, Almuhanna M, et al. A practical approach to the management of cancer patients during the novel coronavirus disease 2019 (COVID-19) pandemic: an international collaborative group. Oncologist. 2020 Jun;25:e936-45.

5 Omboni S. Telemedicine during the COVID-19 in Italy: a missed opportunity? Telemed J E Health. 2020 Aug;26:973-5.

6 Thiessen M, Soriano AM, Loewen HJ, Decker $\mathrm{KM}$. Impact of telemedicine use by oncology physicians on the patient and informal caregiver experience of receiving care: Protocol for a scoping review in the context of COVID-19. JMIR Res Protoc. 2020 Dec 15;9: e25501.
7 Lee I, Kovarik C, Tejasvi T, Pizarro M, Lipoff JB. Telehealth: helping your patients and practice survive and thrive during the $\mathrm{CO}$ VID-19 crisis with rapid quality implementation. J Am Acad Dermatol. 2020 May;82: 1213-4.

8 Hau YS, Kim JK, Hur J, Chang MC. How about actively using telemedicine during the COVID-19 pandemic? J Med Syst. 2020 Apr 30;44:108.

9 Bryant MS, Fedson SE, Sharafkhaneh A. Using telehealth cardiopulmonary rehabilitation during the COVID-19 pandemic. J Med Syst. 2020 May 28;44:125.

10 Prasad A, Brewster R, Newman JG, Rajasekaran K. Optimizing your telemedicine visit during the COVID-19 pandemic: practice guidelines for patients with head and neck cancer. Head Neck. 2020 Jun;42:1317-21.
11 Sirintrapun SJ, Lopez AM. Telemedicine in cancer care. Am Soc Clin Oncol Educ Book. 2018 May 23;38:540-5.

12 Silvestris N, Di Maio M, Russo A, Chiari R, De Giorgi U, Del Mastro L, et al. COVID-19 infection in cancer patients: what has been the contribution of Associazione Italiana Oncologia Medica (AIOM) to oncological care since the beginning of the first pandemic wave?. ESMO Open. 2021 Apr;6(2):100100.

13 Darcourt JG, Aparicio K, Dorsey PM, Ensor JE, Zsigmond EM, Wong ST, et al. Analysis of the implementation of telehealth visits for care of patients with cancer in Houston during the COVID-19 pandemic. JCO Oncol Pract. 2021 Jan;17:e36-43. 